\title{
Substituting dietary fishmeal with soybean meal isolate influences hepatic lipid metabolism and gut microbiota in spotted seabass (Lateolabrax maculatus)
}

\section{Zhe Wang, Linkun Yang, Chunxiao Zhang, Xueshan Li, Ling Wang, Kangle Lu, Kai Song*}

Key Laboratory for Feed Quality Testing and Safety Evaluation, Fisheries College of Jimei University, Xiamen 361021, China

Key words: soy protein isolate; fishmeal replacement; lipid metabolism; gut microbiota; spotted seabass

\begin{abstract}
This study was conducted to investigate the effects of partial substitution of dietary fishmeal (FM) by soy protein isolate (SPI) on lipid metabolism and gut microbiota of spotted seabass. A diet containing 30\% FM formed the basal diet (FM), and two SPI diets were formulated in which 25\% (SPI25) and 50\% (SPI50) of FM were replaced by SPI. Each diet was fed to triplicates of fish for 56 days. The results showed that replacing dietary FM with SPI reduced triglyceride and cholesterol contents in the liver and serum, and the hepatic lipid droplets area was also decreased by dietary SPI inclusion. Furthermore, replacement of dietary FM with SPI markedly down-regulated the mRNA expression of genes involved in lipogenesis (srebpc-1c, fas, acc1, hmgcr, ppary and chrebp) and lipolysis (atgl, hsl, ppara, and cpt1). Moreover, highthroughput sequencing analyses of gut microbiota revealed that dietary SPI inclusion dramatically decreased the Firmicutes/Bacteroidetes ratio. Overall, this study indicated that replacing $25-50 \%$ of dietary FM with SPI could reduce lipid accumulation in serum and liver of spotted seabass, which was associated with the suppressed hepatic lipogenesis and the remodeled gut microbiota.
\end{abstract}

* Corresponding author. Kai Song, e-mail: songkai@jmu.edu.cn 


\section{Introduction}

Sustainable development of aquaculture depends on identifying viable alternatives to fishmeal (FM). Soy protein isolate (SPI), which is the most refined soy protein product, is produced through aqueous solubilization followed by isoelectric precipitation. Most of the anti-nutritional factors (ANFs) contained in SPI are eliminated during the production process, and the protein contents could be extended to over 90\% (Deng et al., 2009). Accordingly, SPI has shown promise as a fishmeal replacer in the aquaculture industry. Previous studies in fish showed that replacing dietary SPI with FM affected the growth performance, feed utilization, nutrients digestibility, hematological parameters, ect. (Blaufuss and Trushenski, 2012; Nepal et al., 2018; Riche and Williams, 2011; Yaghoubi et al., 2016). In addition, the meta-analyses of human studies revealed that soy protein intake was associated with the decreases in serum triglycerides (TG), cholesterol, and lowdensity lipoprotein cholesterol (LDL-C) (Anderson et al., 1995; Zhan and Ho, 2005). Similarly, Nepal et al. (2018) noted that significantly reduced plasma cholesterol was caused by replacing dietary FM with SPI in common carp (Cyprinus carpio). Also, Riche and Williams (2011) found that the body lipid of Florida pompano (Trachinotus carolinus) dramatically decreased with increasing dietary SPI levels. The above findings indicated the potential lipid-lowering activity of SPI versus animal-origin proteins. However, the mechanisms through which SPI exerts its effects on lipid metabolism in fish have not been well investigated.

Spotted seabass (Lateolabrax maculatus) is a major aquaculture species in Asia due to its high economic value and delicious taste. In recent years, the intensification of aquaculture and high dietary lipid levels favors the occurrence of fatty liver in this species associated with abnormal lipid accumulation (Xie et al., 2021). To date, research concerning the effects of replacing FM with SPI in this species is lacking. Accordingly, the current study aimed to investigate substituting dietary FM with SPI on lipid metabolism and gut microbiota in spotted seabass.

\section{Materials and methods}

This research was approved by the Committee on the Ethics of Animal Experiments of Jimei University (Xiamen, China).

\section{Diet formulation and feeding trial}

Three experimental diets were formulated to contain $44 \%$ crude protein and $11 \%$ crude lipid (Table 1). A basal diet was prepared to include $30 \%$ FM. We designed two other experimental diets prepared by replacing 25 or $50 \%$ of FM with SPI (referred to as FM, SPI25, SPI25, respectively). The procedures of diet formulation were conducted as described in our recent work (Cheng et al., 2021).

Spotted seabass juveniles were obtained from a commercial hatchery (Zhangzhou, China) and transported to the fisheries laboratory of Jimei University. All fish were fed a basal diet for 14 days to become acclimated to the conditions and experimental diets. Then, a total of 225 fish (similar initial weight, $8.75 \pm 0.20 \mathrm{~g}$ ) were distributed into nine 20-L tanks supplied with fresh water at a density of 25 fish per tank. Three tanks were randomly assigned to each group, and fish were hand-fed to apparent satiation twice daily $(8: 00,18: 00)$ for 8 weeks. Almost $35 \%$ of water in each tank was renewed daily. The feeding trial was conducted in the recirculating culture system, and water quality was measured weekly. Generally, the water temperature was maintained at $27.0 \pm 1.0^{\circ} \mathrm{C}$, the dissolved oxygen content was approximately $8.0 \mathrm{mg} / \mathrm{L}$, water $\mathrm{pH}$ was $7.5-8.5$, ammonia was below $0.1 \mathrm{mg} / \mathrm{L}$, and a $12 \mathrm{~h}$ light/12 $\mathrm{h}$ dark photoperiod was maintained with fluorescent lights during the feeding trial. 
Table 1 Formulation and proximate composition of the experimental diets.

\begin{tabular}{|c|c|c|c|}
\hline \multirow[t]{2}{*}{ Ingredients ( $\mathrm{g} / \mathrm{kg})$} & \multicolumn{3}{|c|}{ Diet designation } \\
\hline & FM & SPI25 & SPI50 \\
\hline Brown fishmeal & 300.0 & 225.0 & 150.0 \\
\hline Soy protein isolated & 0.0 & 60.0 & 120.0 \\
\hline Wheat flour & 312.4 & 321.7 & 329.9 \\
\hline Wheat gluten & 130.0 & 130.0 & 130.0 \\
\hline Poultry by-product meal & 80.0 & 80.0 & 80.0 \\
\hline Fish oil & 20.0 & 24.0 & 29.0 \\
\hline Soybean oil & 25.0 & 25.0 & 25.0 \\
\hline Lecithin & 20.0 & 20.0 & 20.0 \\
\hline Squid paste & 20.0 & 20.0 & 20.0 \\
\hline Microcrystalline cellulose & 50.0 & 50.0 & 50.0 \\
\hline Vitamin C & 0.5 & 0.5 & 0.5 \\
\hline Monocalcium phosphate & 20.0 & 20.0 & 20.0 \\
\hline DL-Methionine & 3.2 & 4.2 & 5.3 \\
\hline Lysine monohydrochloride & 4.9 & 5.6 & 6.3 \\
\hline Choline chloride & 5.0 & 5.0 & 5.0 \\
\hline Mineral premix ${ }^{1}$ & 5.0 & 5.0 & 5.0 \\
\hline Vitamin premix ${ }^{2}$ & 3.0 & 3.0 & 3.0 \\
\hline $\mathrm{Y}_{2} \mathrm{O}_{3}$ & 1.0 & 1.0 & 1.0 \\
\hline \multicolumn{4}{|l|}{ Proximate compositions (\%) } \\
\hline Crude protein & 43.68 & 43.74 & 43.97 \\
\hline Crude lipid & 11.07 & 11.21 & 11.29 \\
\hline
\end{tabular}

$\overline{1,2}$ Mineral premix and vitamin premix were prepared according to our recent research on spotted seabass (Cheng et al., 2021).

\section{Sample collection}

At the end of the feeding trial, twelve fish per tank were anesthetized with eugenol (1: 10000). The total length was determined, and fish blood was collected from the caudal vein using a sterile syringe and stored at $4^{\circ} \mathrm{C}$ overnight. Serum samples were then separated by centrifugation $\left(4000 \times g, 10 \mathrm{~min}, 4^{\circ} \mathrm{C}\right)$ and stored at $-80^{\circ} \mathrm{C}$. Liver samples of two fish per tank and fixed into $4 \%$ paraformaldehyde for histology analysis. Livers were collected from additional fish in each tank were sampled for RNA extraction and biochemical 
parameters. Mid intestine samples of two fish in each tank were collected and pooled for microbiota analysis. All analyses were performed in triplicates.

Histology analysis and biochemical parameters

Liver tissues were stained with Oil Red O by Service Biotechnology Co., Ltd. (Wuhan, China) following the standard histological procedures for histology analysis. Besides, liver samples were homogenized in phosphate-buffered saline (PBS) and centrifuged (4000 $\times g$, $10 \mathrm{~min}, 4^{\circ} \mathrm{C}$ ) later. Then, the supernatant was collected and stored at $-80^{\circ} \mathrm{C}$ for subsequent analysis. Liver samples were used to detect the contents of TG, total cholesterol (TC), and total bile acid (TBA), as well as activities of lipoprotein lipase (LPL) and hepatic lipase (HL). Besides, serum samples were used to determine the contents of TC, TG, LDL-C, and highdensity lipoprotein cholesterol (HDL-C). According to the manufacturer's instructions, all the above biochemical parameters were analyzed by colorimetric method with commercial kits (Nanjing Jiancheng Biological Company, China).

\section{Quantitative real-time PCR}

Protocols for hepatic total RNA extraction, RT-PCR, and quantitative PCR were performed according to our established work (Cheng et al., 2021). Primers of genes (srebpc-1c, fas, acc1, hmgcr, ppary, chrebp, atgl, hsl, ppara, and cpt1) were designed with the Primer Premier 5.0 software based on nucleotide sequences of spotted seabass (Table 2). The relative expression levels of genes were calculated according to the comparative Ct method 2- $\triangle \Delta \mathrm{Ct}$ (Livak and Schmittgen, 2001).

Table 2 Sequences of the PCR primers used in this study.

\begin{tabular}{|c|c|c|}
\hline Genes & Forward $\left(5^{\prime}-3^{\prime}\right)$ & Reverse $\left(5^{\prime}-3^{\prime}\right)$ \\
\hline $\operatorname{acc1}$ & AAGGCGGTGGTGATGGATIT & GGCCATGTCGCCTTTGTTTT \\
\hline fas & AAACTGAAGCCCTGTGTGCC & САСССТGССТАТTACATTGСTC \\
\hline srebp-1c & ССТСАСТCTGCAGCCAATCA & CGTAGTCCCACCCTCAAACC \\
\hline ppary & TGTGCGTCTGAAGAAACCCT & TACGTCAACGGCATCGCTAA \\
\hline$h m g c r$ & GACCGTGCATACGGAACAGA & AGTGTGTGGGTTGAGACCG \\
\hline chrebp & GTGACAACGCTCAGCTCTCA & TGATGGCAGAGTTCAGGAGC \\
\hline atgl & СТTCCTCTCCGCAACAAGTC & TGGTGCTGTCTGGAGTGTTC \\
\hline hsl & CGAAACACAGAGACGGTCCA & TCATGACATCTACCAGCCGC \\
\hline ppara & CCGTGCGTGTTTTCACCATT & AGACCAAATACATCGCCCCC \\
\hline cpt1 & ССTCAATGATACATCGGAACCC & CTGCGGCTCATCATCTAACG \\
\hline cyp7a1 & GTGTCGTATCCCCAAAGCGA & GGTCCTGCCGTGTAATCTGT \\
\hline cyp8b1 & AGGAGAACCCCCGTTGGATA & СССTGCCAACTGTATCGTGA \\
\hline$\beta$-actin & CGCCGCACTGGTTGTT & TTTGGGGTTCAGGGGG \\
\hline
\end{tabular}

Abbreviations: acc1, acetyl-CoA carboxylase 1; fas, fatty acid synthase; srebp, sterol regulatory element-binding protein; ppar, proliferator-activated receptor; hmgcr, 3-hydroxy-3-methylglutarylCoA reductase; chrebp, carbohydrate responsive element binding protein; atgl, adipose triglyceride lipase; hsl, hormone sensitive lipase; cpt1, carnitine palmityltransferase 1 ; cyp7a1, cholesterol 7a hydroxylase; cyp $8 b 1$, sterol 12a-hydroxylase. 


\section{Gut microbiota analysis}

Total gut bacterial DNA was extracted with a HiPure Soil DNA Kit (Magen, Guangzhou, China). The sequence analyses of the V3-V4 region of 16S rRNA gene were performed by Gene Denovo Biotechnology Co., Ltd. (Guangzhou, China) using Illumina MiSeq PE 250.

\section{Statistical analysis}

The homogeneity of variances of data was identified, and data were then analyzed using one-way ANOVA followed by Tukey's test (SPSS 22.0). Statistical significance was set at $P<0.05$, and results were presented as mean \pm SEM of triplicate groups.

\section{Liver histology and biochemical parameters}

\section{Results}

Oil red $\mathrm{O}$ staining results indicated the accumulation of liver lipid droplets decreased with dietary SPI inclusion (Figure 1). Serum TG, TC, and LDL-C contents were markedly decreased by replacing $50 \%$ of dietary FM with SPI $(P<0.05)$ (Table 3 ), while these traits did not differ between FM and SPI25 groups $(P>0.05)$. No significant difference in serum HDL-C content was observed among all groups $(P>0.05)$. Hepatic TG and TC contents significantly decreased with the increase of dietary SPI levels $(P<0.05)$ (Table 4), while an opposing trend was detected in $\mathrm{HL}$ activity among all treatments $(P<0.05)$. No significant difference in hepatic TBA content and LPL activity was detected among all groups $(P>0.05)$. These results indicated that substituting dietary FM with SPI reduced lipid accumulation in spotted seabass.
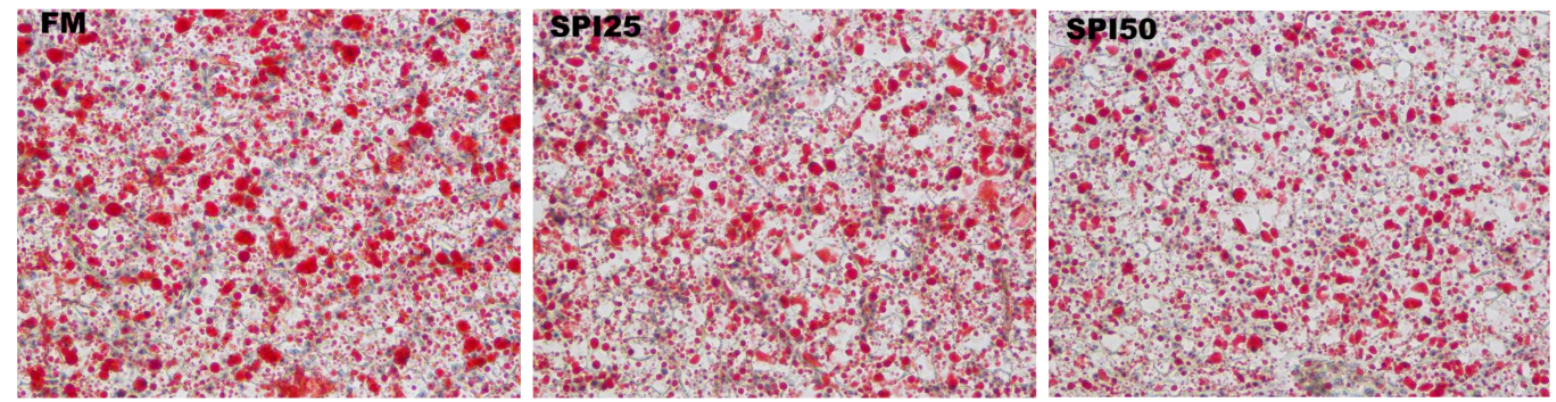

Figure 1 Oil O red staining sections of liver for spotted seabass fed experimental diets for 8 weeks (scale bar $=100 \mu \mathrm{m})$.

Table 3 Serum biochemical indexes ( $\mathrm{mmol} / \mathrm{L}$ ) of spotted seabass fed experimental diets for 8 weeks.

\begin{tabular}{llll}
\hline Items & \multicolumn{3}{l}{ Experimental diet treatments } \\
\cline { 2 - 4 } & FM & SPI25 & SPI50 \\
\hline $\mathrm{TG}^{1}$ & $5.72 \pm 0.11^{\mathrm{b}}$ & $5.39 \pm 0.13^{\mathrm{b}}$ & $4.14 \pm 0.08^{\mathrm{a}}$ \\
$\mathrm{TC}^{2}$ & $17.08 \pm 0.17^{\mathrm{b}}$ & $15.87 \pm 0.64^{\mathrm{b}}$ & $13.04 \pm 0.18^{\mathrm{a}}$ \\
LDL-C $^{3}$ & $5.14 \pm 0.53^{\mathrm{b}}$ & $4.51 \pm 0.11^{\mathrm{b}}$ & $3.40 \pm 0.11^{\mathrm{a}}$ \\
$\mathrm{HDL}-C^{4}$ & $6.66 \pm 0.13$ & $6.13 \pm 0.13$ & $5.73 \pm 0.35$
\end{tabular}

Values are mean \pm SEM of three groups of fish. Values in the same row with different superscripts are significantly different $(P<0.05)$.

${ }^{1} \mathrm{TG}$ : triglyceride.

2 TC: total cholesterol.

3 LDL-C: low-density lipoprotein cholesterol.

${ }^{4}$ HDL-C: high-density lipoprotein cholesterol. 
Table 4 Hepatic biochemical indexes of spotted seabass fed experimental diets for 8 weeks.

\begin{tabular}{|c|c|c|c|}
\hline \multirow[t]{2}{*}{ Items } & \multicolumn{3}{|c|}{ Experimental diet treatments } \\
\hline & $\mathrm{FM}$ & SPI25 & SPI50 \\
\hline $\mathrm{TG}^{1}$ & $14.85 \pm 0.54^{c}$ & $12.99 \pm 0.10^{\mathrm{b}}$ & $11.35 \pm 0.46^{\circ}$ \\
\hline $\mathrm{TC}^{2}$ & $2.08 \pm 0.15^{b}$ & $1.88 \pm 0.08^{\mathrm{ab}}$ & $1.69 \pm 0.02^{\mathrm{a}}$ \\
\hline TBA $^{3}$ & $32.29 \pm 0.07$ & $33.06 \pm 0.04$ & $33.73 \pm 0.54$ \\
\hline $\mathrm{LPL}^{4}$ & $4.01 \pm 0.01$ & $4.55 \pm 0.17$ & $4.68 \pm 0.31$ \\
\hline $\mathrm{HL}^{5}$ & $3.66 \pm 0.16^{a}$ & $3.62 \pm 0.33^{a}$ & $4.34 \pm 0.11^{b}$ \\
\hline
\end{tabular}

Values are mean \pm SEM of three groups of fish. Values in the same row with different superscripts are significantly different $(P<0.05)$.

${ }^{1}$ TG: triglyceride (mmol/gprot).

2 TC: total cholesterol ( $\mathrm{mmol} / \mathrm{gprot})$.

3 TBA: total bile acid (mmol/gprot).

${ }^{4}$ LPL: lipoprotein lipase (U/mgprot).

${ }^{5} \mathrm{HL}$ : hepatic lipase (U/mgprot).

Relative mRNA expression of genes involved in lipid and bile acid metabolism

The expressions of lipid synthesis-related genes including srebpc-1c, fas, acc1, hmgcr, ppary and chrebp dramatically decreased by replacing dietary FM with SPI $(P<0.05)$ (Figure 2A). Consistently, dietary SPI inclusion also down-regulated the expressions of genes involved in lipid catabolism, including atgl, hsl, ppara, and cpt1 $(P<0.05)$ (Figure 2B). No marked differences in bile acid synthesis-related genes cyp7a1 and cyp8b1 were observed among all treatments $(P>0.05)$ (Figure 2C). These results indicated that substituting dietary FM with SPI suppressed lipid synthesis and catabolism in spotted seabass. 

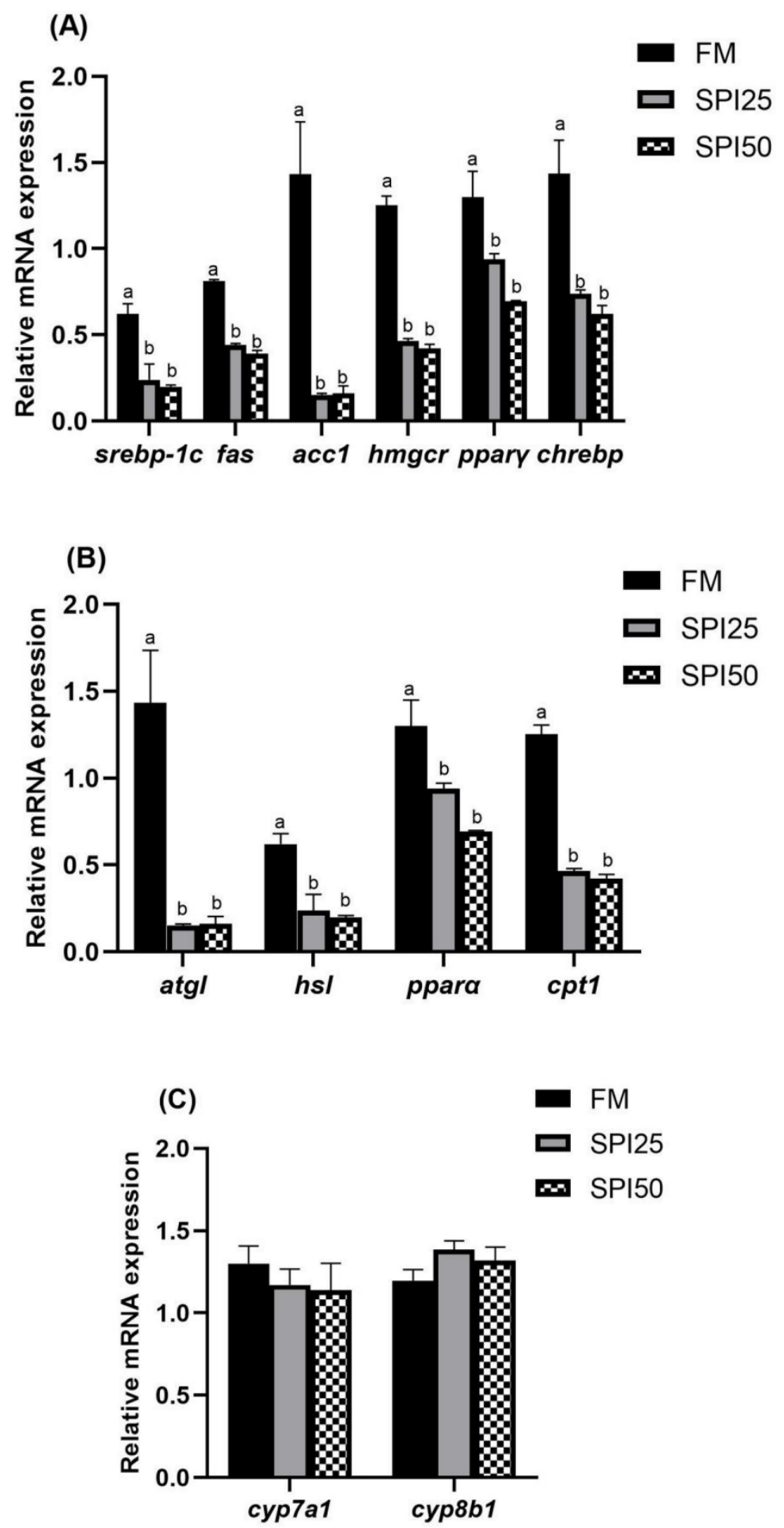

Figure 2 Relative mRNA expression of genes involved in (A) Lipid synthesis, (B) Lipid catabolism, and $(C)$ Bile acid synthesis in the liver of spotted seabass fed experimental diets for 8 weeks. Bars with different letters are significantly different $(P<0.05)$. 


\section{Gut microbiota}

Venn diagram of bacterial communities showed that the core OTUs among all groups was 45, and independent OTUs of FM, SPI25, and SPI50 groups were 601, 383, and 451, respectively (Figure 3A). Alpha-diversity indexes including ACE, Chao1, Shannon and Simpson were shown in Table 5. It could be observed that ACE and Chao1 indexes dramatically increased with the increase in dietary SPI levels $(P<0.05)$, while Shannon and Simpson's indexes did not affect $(P>0.05)$.

In general, Proteobacteria, Bacteroidetes, and Firmicutes were the three major bacterial phyla in the mid intestinal of spotted seabass (Figure 3B). The Proteobacteria abundance in the FM group was significantly higher than that in both SPI25 and SPI50 groups $(P<0.05)$ (Figure 3D), and the abundance of Bacteroidetes $(P<0.05)$ and Firmicutes $(P>0.05)$ increased along with dietary SPI inclusion. The dominant genus in FM and two SPI groups was Plesiomonas and Prevotella, respectively (Figure 3C). Replacing dietary FM with SPI significantly increased the abundance of Prevotella, Succiniclasticum, and Desulfovibrio in the gut of spotted seabass $(P<0.05)$ (Figure 3E). These results indicated that substituting dietary FM with SPI changed gut microbiota profile in spotted seabass.
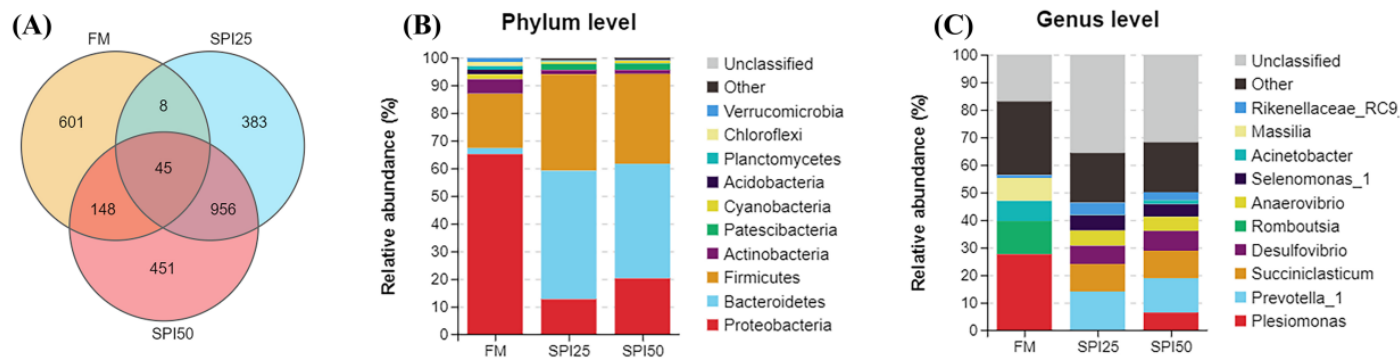

(D)

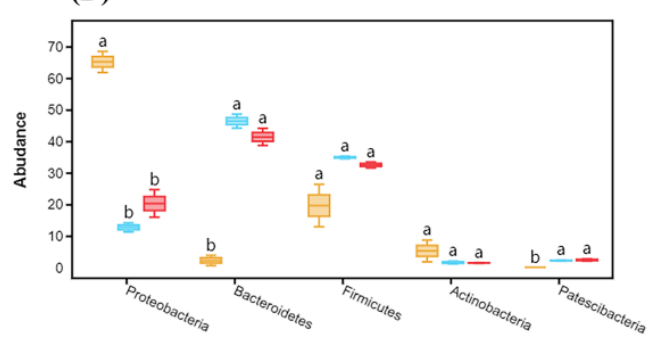

(E)

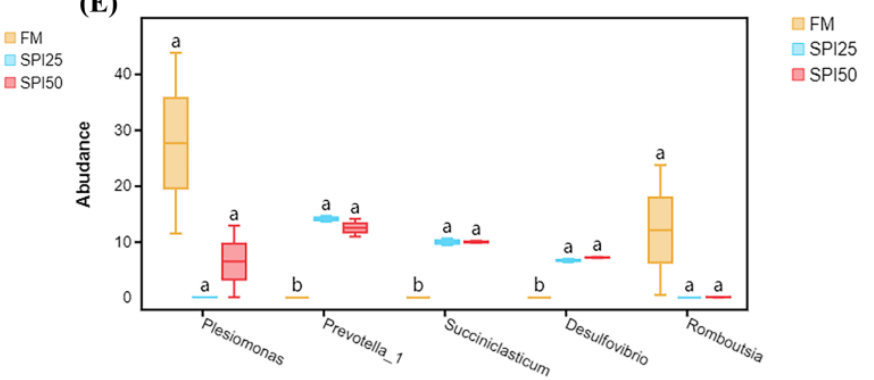

Figure 3 16S rRNA gene sequencing analysis of the gut microbiota. (A) Venn diagram; Percent of community abundance at (B) phylum and (C) genus level; The relative abundance of major bacterial (D) phyla and (E) genera in boxplot; Bars with different letters are significantly different $(P<0.05)$. 
Table 5 Alpha-diversity indexes (including ACE, Chao1, Shannon and Simpson) of spotted seabass fed experimental diets for 8 weeks.

\begin{tabular}{|c|c|c|c|}
\hline \multirow[t]{2}{*}{ Items } & \multicolumn{3}{|c|}{ Experimental diet treatments } \\
\hline & FM & SPI25 & SPI50 \\
\hline ACE & $967.09 \pm 33.35^{a}$ & $2239.01 \pm 59.14^{b}$ & $2540.91 \pm 88.42^{c}$ \\
\hline Chao1 & $936.76 \pm 50.32^{a}$ & $2170.06 \pm 21.06^{b}$ & $2409.72 \pm 119.31^{b}$ \\
\hline Shannon & $5.33 \pm 1.04$ & $7.14 \pm 0.19$ & $7.06 \pm 0.11$ \\
\hline Simpson & $0.90 \pm 0.08$ & $0.99 \pm 0.00$ & $0.98 \pm 0.00$ \\
\hline Coverage (\%) & $99.77 \pm 0.03^{b}$ & $99.38 \pm 0.00^{a}$ & $99.33 \pm 0.04^{a}$ \\
\hline
\end{tabular}

Values are mean \pm SEM of three groups of fish. Values in the same row with different superscripts are significantly different $(P<0.05)$.

\section{Discussion}

Plant-derived protein has been proven to exert hypolipidemic effects on animals in comparison to animal protein sources (Torres et al., 2006; Ijaz et al., 2018). In the present study, substituting dietary FM with SPI led to reduced lipid droplets area, TG, and TC contents in both serum and liver. Besides, $\mathrm{HL}$, a key hydrolytic enzyme of TG, decreased along with dietary SPI inclusion. These results revealed that substitution of dietary FM with SPI could reduce lipid accumulation in spotted seabass. Similar results were also observed in Japanese flounder (Paralichthys olivaceus) (Deng et al., 2009). Research on rats showed that soy protein participated in lipid homeostasis by regulating several transcription factors such as the srebp family (Torres et al., 2006). The srebp 1 isoform was preferentially involved in de novo lipogenesis of fatty acids via activating lipogenic genes, fas and acc, required for lipid synthesis; whereas srebp2 isoform controls the cholesterol homeostasis by activating hmgcr gene, required for cholesterol synthesis (Dihingia et al., 2018). Besides, ppary was proven to regulate adipocyte differentiation and adipocyte hypertrophy (Li et al., 2018). In the current study, replacing dietary FM with SPI resulted in lower mRNA expressions of srebp1, fas, acc1, hmgcr, and ppary. A generally accepted biochemical principle is that energy metabolism is coordinately regulated by multiple pathways (Sharpe and Brown, 2013). chrebp was a key factor in converting glucose to TG for storage, and it was demonstrated that chrebp and srebp factors could act in synergy to induce transcription of lipogenic genes (Dentin et al., 2005). In the present study, the expressions of both chrebp and srebp-1c genes decreased along with dietary SPI inclusion. Therefore, it could be concluded that the substitution of dietary FM with SPI suppressed the hepatic lipogenesis of spotted seabass.

Besides lipogenesis, lipolysis also played a crucial role in lipid metabolism for animals (Peng et al., 2014). atgl and hsl, two major lipid hydrolases, were proven to directly regulate lipolysis in a step-wise approach. More specifically, the lipolysis was initiated by atgl via cleaving one fatty acid from TG, and hsl then acted on diacylglycerol releasing two additional fatty acids and one glycerol molecule (Jin et al., 2017). Additionally, the oxidation of fatty acids, referred to as $\beta$-oxidation, was regulated by ppara and cpt1 (a crucial rate-limiting enzyme of $\beta$-oxidation) (Lai et al., 2021). The information about the role of SPI in the regulation of hepatic lipolysis is limited. Research on rats indicated that soy protein-derived peptide simulated fat oxidation via activating lipolysis-related transcription factors (Torres et al., 2006). In contradiction to the above result, research on 
fish showed that replacing dietary FM with plant-protein source suppressed the expression of ppara and cpt1, and the LPL activity in the liver (Ye et al., 2019). Consistently, the current study revealed that replacing dietary FM with SPI down-regulated the expression of lipolysis-related genes including ppara, cpt1, atgl, and $h s l$. It might be due to that the liver lipolysis was suppressed via a feedback regulatory mechanism in response to a lowfat statue of SPI-fed fish, thus achieving a dynamic equilibrium between lipid synthesis and catabolism. In line with this point, previous studies demonstrated the expression of aforementioned genes (ppara, cpt1, and atgl) increased along with hepatic lipid contents (Gou et al., 2019). Moreover, the regulation mechanisms of lipid metabolism vary from terrestrial and fish, and it should be investigated on a case-by-case basis.

A previous study revealed that SPI-derived 7S protein involved cholesterol metabolism via stimulating bile acid synthesis (Liu et al., 2017). Thus, we determined the TBA content and expression of cyp7a1 and cyp8a1 in the liver. However, no significant difference was obtained in these traits among all groups. Replacing dietary FM with SPI suppressed lipolysis and lipogenesis in the liver indicated that a systematic fatty acid cycle was inactive in SPI-fed fish versus FM-fed fish. In addition, Lipids synthesis, catabolism, and transport were generally regulated by controlling key enzymes through allosteric and covalent modifications (Dentin et al., 2005). Thus, the lipid metabolism-related enzymes still need further investigation at the post-translational level.

Diet intake establishes a central determinant of the compositions of trillions of microorganisms residing in the gut. Furthermore, diet-induced modulation of gut microbiota directly influences the host physiological state (Xu et al., 2021; Wang et al., 2021). Thus, it is imperative to understand how protein intake from different sources affects gut microbiota. Soy protein was proven to have particular potential in increasing the gut microbial diversity in animals (Butteiger et al., 2016). The current study showed that substitution of dietary FM with SPI led to high microbial diversity and richness, which ACE and chao1 indexes indicated. The gut microbiota compositions have also been remolded by dietary SPI inclusion. Specifically, The SPI-fed group had a relatively higher abundance of Bacteroidetes and Firmicutes than the FM-fed group, which was dominant by Proteobacteria. Consistently, Ingerslev et al. (2014) reported that different dietary types determined gut microbiota community in rainbow trout (Oncorhynchus mykiss). Firmicutes was dominant in the gut of plant based-fed fish, whereas Proteobacteria in marine basedfed fish. The Firmicutes/Bacteroidetes (F/B) ratio was usually used to quantify the gut microbiota of obese and lean individuals. A high proportion of $F / B$ resulted in more effective energy absorption and subsequent weight gain (Kong et al., 2021). A previous study showed that replacing $20 \%$ of dietary animal protein with soy protein for 6 weeks increased the F/B ratio in fecal microbiota (Lee et al., 2015). Similarly, the present study showed a markedly lower F/B ratio in both SPI25 and SPI50 groups ( 0.75 and 0.78$)$ compared with the FM group (8.94). This observation was supported by the lean phenotype of SPI-fed fish versus FM-fed fish.

A bloom of the Prevotella genus characterized the SPI-fed group. This species was reported to negatively correlate with obesity and metabolic disorders in numerous studies (Arnoriaga-Rodriguez et al., 2020). Nutritional interventions with fiber-rich foods usually led to an increased Prevotella abundance, which was capable of breaking down dietaryderived plant polysaccharides but not animal polysaccharides (Tett et al., 2021). In addition, SPI-fed fish were unique in showing a greater abundance of Desulfovibrio. It has been reported that $D$. Vulgaris within Desulfovibrio spp. could prevent fatty liver disease by inhibiting fatty acid de novo synthesis (Hong et al., 2021). Accordingly, the alterations in spotted seabass's lipid metabolism patterns partially resulted from the remodeled gut microbiota caused by dietary SPI inclusion.

In conclusion, results of the present study showed that replacing $25-50 \%$ of dietary FM with SPI reduced lipid accumulation in the serum and liver of spotted seabass, which was associated with the down-regulation of genes involved in lipolysis and lipogenesis, as well as the alterations in gut microbiota. 


\section{Acknowledgments}

This study was supported by the Natural Science Foundation of Fujian Province of China (grant number: 2019J01060380) and the China Agriculture Research System (grant number: CARS47-14). We thank Shi Cao for his valuable help in sample analyses.

\section{References}

Arnoriaga-Rodriguez M., Mayneris-Perxachs J., Burokas A., Perez-Brocal V., Moya A., Portero-Otin M., and Fernandez-Real J.M., 2020. Gut bacterial ClpB-like gene function is associated with decreased body weight and a characteristic microbiota profile. Microbiome, 8(1): 59. doi:10.1186/s40168-020-00837-6

Blaufuss P. and Trushenski J., 2012. Exploring Soy-Derived Alternatives to Fishmeal: Using Soy Protein Concentrate and Soy Protein Isolate in Hybrid Striped Bass Feeds. N. Am. J. Aquacult., 74(1): 8-19. doi:10.1080/15222055.2011.635782

Butteiger D.N., Hibberd A.A., McGraw N.J., Napawan N., Hall-Porter J.M., and Krul E.S., 2016. Soy Protein Compared with Milk Protein in a Western Diet Increases Gut Microbial Diversity and Reduces Serum Lipids in Golden Syrian Hamsters. J. Nutr., 146(4): 697-705. doi:10.3945/jn.115.224196

Cheng Y., Li X., Wang L., Lu K., Song K., Ai Q., Mai K., and Zhang C., 2021. Effects of dietary arginine levels on growth, immune function of physical barriers and serum parameters of spotted seabass (Lateolabrax maculatus) reared at different water temperatures. Aquaculture, 541: 736812 doi:10.1016/j.aquaculture.2021.736812

Deng J., Mai K., Ai Q., Zhang W., Wang X., Tan B., Xu W., Liu Z., and Ma H., 2009. Interactive effects of dietary cholesterol and protein sources on growth performance and cholesterol metabolism of Japanese flounder (Paralichthys olivaceus). Aquacult. Nutr., 16(4): 419-429. doi:10.1111/j.1365-2095.2009.00681.x

Dentin R., Girard J., and Postic C., 2005. Carbohydrate responsive element binding protein (ChREBP) and sterol regulatory element binding protein-1c (SREBP-1c): two key regulators of glucose metabolism and lipid synthesis in liver. Biochimie, 87(1): 81-86. doi:10.1016/j.biochi.2004.11.008

Dihingia A., Bordoloi J., Dutta P., Kalita J., and Manna P., 2018. HexaneIsopropanolic Extract of Tungrymbai, a North-East Indian fermented soybean food prevents hepatic steatosis via regulating AMPK-mediated SREBP/FAS/ACC/HMGCR and PPARalpha/CPT1A/UCP2 pathways. Sci. Rep., 8(1): 10021. doi:10.1038/s41598-018-27607-7

Gou N., Chang Z., Deng W., Ji H., and Zhou J., 2019. Effects of dietary lipid levels on growth, fatty acid composition, antioxidant status and lipid metabolism in juvenile Onychostoma macrolepis. Aquac. Res., 50(11), 3369-3381. doi:10.1111/are.14295

Hong Y., Sheng L., Zhong J., Tao X., Zhu W., Ma J., Yan J., Zhao A., Zheng X., Wu G., Li B., Han B., Ding K., Zheng N., Jia W., and Li H., 2021. Desulfovibrio vulgaris, a potent acetic acid-producing bacterium, attenuates nonalcoholic fatty liver disease in mice. Gut Microbes, 13(1), 1-20. doi:10.1080/19490976.2021.1930874

Ijaz M.U., Ahmed M.I., Zou X., Hussain M., Zhang M., Zhao F., Xu X., Zhou G, and Li C., 2018. Beef, Casein, and Soy Proteins Differentially Affect Lipid Metabolism, Triglycerides Accumulation and Gut Microbiota of High-Fat Diet-Fed C57BL/6] Mice. Front. Microbiol., 9: 2200. doi:10.3389/fmicb.2018.02200

Ingerslev H.C., Gersdorff Jørgensen L., Lenz Strube M., Larsen N., Dalsgaard I., Boye M., and Madsen L., 2014. The development of the gut microbiota in rainbow trout (Oncorhynchus mykiss) is affected by first feeding and diet type. Aquaculture, 424-425: 24-34. doi:10.1016/j.aquaculture.2013.12.032

Jin M., Yuan Y., Lu Y., Ma H., Sun P., Li Y., Qiu H., Ding L., and Zhou Q., 2017. Regulation of growth, tissue fatty acid composition, biochemical parameters and lipid related genes expression by different dietary lipid sources in juvenile black seabream, Acanthopagrus schlegelii. Aquaculture, 479: 25-37. doi:10.1016/j.aquaculture.2017.05.017 
Kong Y., Liao Z., Ma X., Liang M., Xu H., Mai K., and Zhang Y., 2021. Effects of different dietary lipid levels on intestinal mucosal barrier and microbial community of juvenile tiger puffer Takifugu rubripes. Aquacult. Nutr., 00: 1-14. doi:10.1111/anu.13302

Lai W., Xu D., Li J., Wang Z., Ding Y., Wang X., Li X., Xu N., Mai K., and Ai Q., 2021. Dietary polystyrene nanoplastics exposure alters liver lipid metabolism and muscle nutritional quality in carnivorous marine fish large yellow croaker (Larimichthys crocea). J. Hazard. Mater., 419: 126454. doi:10.1016/j.jhazmat.2021.126454

Lee S.M., Han H.W., and Yim S.Y., 2015. Beneficial effects of soy milk and fiber on high cholesterol diet-induced alteration of gut microbiota and inflammatory gene expression in rats. Food Funct., 6(2): 492-500. doi:10.1039/c4fo00731j

Li D., Ikaga R., and Yamazaki T., 2018. Soya protein beta-conglycinin ameliorates fatty liver and obesity in diet-induced obese mice through the down-regulation of PPARgamma. Brit. J. Nutr., 119(11): 1220-1232. doi:10.1017/S0007114518000739

Liu Y., Yang J., Lei L., Wang L., Wang X., Ma K., Yang X., and Chen Z.Y., 2017. 7 S protein is more effective than total soybean protein isolate in reducing plasma cholesterol. J. Funct. Foods, 36: 18-26. doi:10.1016/j.jff.2017.06.039

Livak K.J., and Schmittgen T.D., 2001. Analysis of relative gene expression data using real-time quantitative PCR and the 2 (-Delta Delta C(T)) method. Methods, 25(4): 402-408. doi: $10.1006 /$ meth.2001.1262

Nepal S., Kumar V., Makkar H.P.S., Stadtlander T., Romano N., and Becker K., 2018. Comparative nutritional value of Jatropha curcas protein isolate and soy protein isolate in common carp. Fish Physiol. Biochem., 44(1): 143-162. doi:10.1007/s10695-017-0420-X

Peng M., Xu W., Mai K., Zhou H., Zhang Y., Liufu Z., Zhang K., and Ai Q., 2014. Growth performance, lipid deposition and hepatic lipid metabolism related gene expression in juvenile turbot (Scophthalmus maximus L.) fed diets with various fish oil substitution levels by soybean oil. Aquaculture, 433: 442-449. doi:10.1016/j.aquaculture.2014.07.005

Riche M. and Williams T.N., 2011. Fishmeal replacement with solvent-extracted soybean meal or soy protein isolate in a practical diet formulation for Florida pompano (Trachinotus carolinus, L.) reared in low salinity. Aquacult. Nutr., 17(4): 368-379. doi:10.1111/j.1365-2095.2010.00808.x

Sharpe L.J. and Brown A.J., 2013. Controlling cholesterol synthesis beyond 3-hydroxy3-methylglutaryl-CoA reductase (HMGCR). J. Biol. Chem., 288(26): 18707-18715. doi:10.1074/jbc.R113.479808

Tett A., Pasolli E., Masetti G., Ercolini D., and Segata N., 2021. Prevotella diversity, niches and interactions with the human host. Nat. Rev. Microbiol., 19(9): 585-599. doi:10.1038/s41579-021-00559-y

Torres N., Torre-Villalvazo I., and Tovar A.R., 2006. Regulation of lipid metabolism by soy protein and its implication in diseases mediated by lipid disorders. J. Nutr. Biochem., 17(6): 365-373. doi:10.1016/j.jnutbio.2005.11.005

Wang T., Zhang N., Yu X.B., Qiao F., Chen L.Q., Du Z.Y., and Zhang M.L., 2021. Inulin alleviates adverse metabolic syndrome and regulates intestinal microbiota composition in Nile tilapia (Oreochromis niloticus) fed with high-carbohydrate diet. Brit. J. Nutr., 126(2): 161-171. doi: 10.1017/s000711452000402x.

Xie S., Yin Y., Wu T., Tian L., Liang J., and Tan B., 2021. Dietary lipid levels affected growth performance, lipid accumulation, inflammatory response and apoptosis of japanese seabass (lateolabrax japonicus). Aquacult. Nutr. 27: 807-816. doi: 10.1111/anu.13225

Xu R., Li M., Wang T., Zhao Y., Shan C., Qiao F., Chen L., Zhang W., Du Z., and Zhang M., 2021. Bacillus amyloliquefaciens ameliorates high-carbohydrate dietinduced metabolic phenotypes by restoration of intestinal acetate-producing bacteria in Nile Tilapia. Brit. J. Nutr., 1-13. doi:10.1017/S0007114521001318 
Yaghoubi M., Yaghoubi M., Mozanzadeh M.T., Marammazi J.G., Safari O., and Gisbert E., 2016. Dietary replacement of fishmeal by soy products (soybean meal and isolated soy protein) in silvery-black porgy juveniles (Sparidentex hasta). Aquaculture, 464: 50-59. doi:10.1016/j.aquaculture.2016.06.002

Ye H., Xu M., Chen L., Tan X., Chen S., Zou C., Sun Z., Liu Q., Ye C., and Wang A., 2019. Effects of dietary plant protein sources influencing hepatic lipid metabolism and hepatocyte apoptosis in hybrid grouper (Epinephelus lanceolatus ơ $\times$ Epinephelus fuscoguttatus 9 ). Aquaculture, 506: 437-444. doi:10.1016/j.aquaculture.2019.03.075 